\title{
A generalized Isserlis theorem for location mixtures of Gaussian random vectors
}

\author{
C.Vignat* \\ E.P.F.L., L.T.H.I., Lausanne, Switzerland
}

\begin{abstract}
In a recent paper, Michalowicz et al. provide an extension of Isserlis theorem to the case of a Bernoulli location mixture of a Gaussian vector. We extend here this result to the case of any location mixture of Gaussian vector; we also provide an example of the Isserlis theorem for a "scale location" mixture of Gaussian, namely the $d$-dimensional generalized hyperbolic distribution.
\end{abstract}

Keywords: Isserlis theorem, normal-variance mixture, generalized hyperbolic distribution

\section{Introduction}

Isserlis theorem, as discovered by Isserlis [1] in 1918, allows to express the expectation of a monomial in an arbitrary number of components of a zero mean Gaussian vector $X \in \mathbb{R}^{d}$ in terms of the entries of its covariance matrix only. Before providing in Thm 1 the slightly generalized version of Isserlis theorem due to Withers [3], we introduce the following notations: for any set $A=\left\{\alpha_{1}, \ldots, \alpha_{N}\right\}$ of integers such that $1 \leq \alpha_{i} \leq d$ and any vector $X \in \mathbb{R}^{d}$, we use the multi-index notation and denote

$$
X_{A}=\prod_{\alpha_{i} \in A} X_{\alpha_{i}}
$$

with the convention that for the empty set

$$
X_{\emptyset}=1 .
$$

A pairing in a set $A$ is a partition of $A$ into disjoint pairs. We denote by $\Pi(A)$ the set of all pairings $\sigma$ in $A$ : note that $\Pi(A)$ is empty if $A$ has an odd number of elements. For a given $\sigma \in \Pi(A)$, we denote by $A / \sigma$ the set $\{i ; \sigma=(i, \sigma(i))\}$; finally, $\sum_{A} \prod \mathbb{E}\left(X_{i} X_{j}\right)$ denotes the sum

$$
\sum_{\sigma \in \Pi(A)} \prod_{i \in A / \sigma} \mathbb{E}\left(X_{\alpha_{i}} X_{\alpha_{\sigma(i)}}\right) .
$$

In other words, for a given pairing $\sigma$ in the set $A$, we compute the product of all possible moments $\mathbb{E}\left(X_{i} X_{j}\right)$ where $i$ and $j$ are paired by $\sigma$; then, $\sum_{A} \mathbb{E}\left(X_{i} X_{j}\right)$ denotes the sum of these products

\footnotetext{
* on leave from L.S.S., Supélec, Orsay, France

Email address: christophe.vignat@epfl.ch (C.Vignat)
} 
over all possible pairings in $A$. As an example

$$
\sum_{\{1,1,2,4\}} \prod_{\mathbb{E}}\left(X_{i} X_{j}\right)=\mathbb{E}\left(X_{1}^{2}\right) \mathbb{E}\left(X_{2} X_{4}\right)+2 \mathbb{E}\left(X_{1} X_{2}\right) \mathbb{E}\left(X_{1} X_{4}\right)
$$

A general form of Isserlis theorem, due to Withers, is as follows.

Theorem 1. If $A=\left\{\alpha_{1}, \ldots, \alpha_{2 N}\right\}$ is a set of integers such that $1 \leq \alpha_{i} \leq d, \forall i \in[1,2 N]$ and $X \in \mathbb{R}^{d}$ is a Gaussian vector with zero mean then

$$
\mathbb{E} X_{A}=\sum \prod_{A} \mathbb{E}\left(X_{i} X_{j}\right)
$$

Moreover, if $A=\left\{\alpha_{1}, \ldots, \alpha_{2 N+1}\right\}$ then, under the same assumptions,

$$
\mathbb{E} X_{A}=0 \text {. }
$$

For example, choosing $\alpha_{i}=i, 1 \leq i \leq 4$ yields the well-known identity

$$
\mathbb{E}\left(X_{1} X_{2} X_{3} X_{4}\right)=\mathbb{E}\left(X_{1} X_{2}\right) \mathbb{E}\left(X_{3} X_{4}\right)+\mathbb{E}\left(X_{1} X_{3}\right) \mathbb{E}\left(X_{2} X_{4}\right)+\mathbb{E}\left(X_{1} X_{4}\right) \mathbb{E}\left(X_{2} X_{3}\right) .
$$

However, indices $\alpha_{i}$ need not be distinct: for example, choosing $\alpha_{i}=1,1 \leq i \leq 4$ yields

$$
\mathbb{E} X_{1}^{4}=3 \mathbb{E} X_{1}^{2}
$$

Several extensions of this result have been provided recently: in [3], Withers extends Isserlis theorem to the case of noncentral Gaussian vectors and relates the result with multivariate Hermite polynomials; in [4], a general formula for Gaussian scale mixtures, and more generally for elliptically distributed vectors is derived; it is applied to the computation of moments of the uniform distribution on the sphere. In [5], Isserlis theorem is extended to the computation of the moments of linear combinations of independent Student-t vectors. In [6], Isserlis theorem is extended to Gaussian matrix mixtures, i.e. random vectors of the form

$$
X=A N
$$

where $N$ is a standard Gaussian vector in $\mathbb{R}^{d}$ and $A$ is a $(d \times d)$ random matrix. Let us also mention the reference [8] where the author tackles the computational complexity of formula (1), using Magnus lemma to replace a product of $n$ variables by sums of polynomials of degree $n$ in these variables.

Recently, Michalowicz et al. [2] addressed the case of Gaussian location mixtures: they provided an extension of Isserlis theorem to the case of a random vector $X \in \mathbb{R}^{d}$ with probability density

$$
f_{X}(x)=\frac{1}{2} \phi_{R}(x+\mu)+\frac{1}{2} \phi_{R}(x-\mu)
$$

where

$$
\phi_{R}(x)=\frac{1}{|2 \pi R|^{\frac{1}{2}}} \exp \left(-\frac{1}{2} x^{t} R^{-1} x\right)
$$

is the $d$-variate Gaussian density with zero mean and covariance matrix $R$.

In the following, we give a new and simple proof of the result by Michalowicz et al., adopting a formalism that allows us to extend their results to the general case of an arbitrary Gaussian location mixture. We also provide an extension of these results to the case of a scale-location mixture of Gaussian. 


\section{Extensions of the result by Michalowicz et al.}

A key observation is that the random vector $X$ with density (2) reads

$$
X=\epsilon \mu+\zeta
$$

where $\epsilon$ is a Bernoulli random variable $\left(\operatorname{Pr}\{\epsilon=-1\}=\operatorname{Pr}\{\epsilon=1\}=\frac{1}{2}\right), \zeta \in \mathbb{R}^{d}$ is a zero mean Gaussian vector and equality is in the sense of distributions. This stochastic representation allows to prove easily a generalized version of the main result of [2], namely

Theorem 2. If $X \in \mathbb{R}^{d}$ is distributed according to (2) and $A=\left\{\alpha_{1}, \ldots, \alpha_{2 N}\right\}$ with $1 \leq \alpha_{i} \leq d$ then

$$
\mathbb{E} X_{A}=\sum_{k=0}^{N} \sum_{\substack{S \subset A \\|S|=2 k}} \mu_{S} \sum_{A \backslash S} \prod_{\mathbb{S}} \mathbb{E}\left(\zeta_{i} \zeta_{j}\right) .
$$

If $A=\left\{\alpha_{1}, \ldots, \alpha_{2 N+1}\right\}$ then $\mathbb{E} X_{A}=0$.

The simplified proof we propose is as follows: by (3),

$$
\mathbb{E} X_{A}=\mathbb{E}(\epsilon \mu+N)_{A}
$$

and since the product $(a+b)_{A}$ can be expanded as

$$
(a+b)_{A}=\sum_{k=0}^{2 N} \sum_{\substack{S \subset A \\|S|=k}} a_{S} b_{A \backslash S},
$$

we deduce that

$$
\mathbb{E} X_{A}=\sum_{k=0}^{2 N} \sum_{\substack{S \subset A \\|S|=k}} \mu_{S} \mathbb{E}\left(\epsilon^{k}\right) \mathbb{E}\left(\zeta_{A \backslash S}\right) .
$$

By Isserlis theorem, the expectation of the product of an odd number of centered Gaussian random variables $\zeta_{i}$ is equal to zero so that this expression simplifies to

$$
\sum_{k=0}^{N} \sum_{\substack{S \subset A \\|S|=2 k}} \mu_{S} \mathbb{E}\left(\epsilon^{2 k}\right) \mathbb{E}\left(\zeta_{A \backslash S}\right) .
$$

Since $\epsilon$ is Bernoulli distributed, all its even moments are equal to 1 ; moreover, since $\zeta$ has zero mean, by Isserlis theorem, $E\left(\zeta_{A \backslash S}\right)=\sum_{A \backslash S} E\left(\zeta_{i} \zeta_{j}\right)$ and we obtain

$$
\mathbb{E} X_{A}=\sum_{k=0}^{N} \sum_{\substack{S \subset A \\|S|=2 k}} \mu_{S} \sum_{A \backslash S} \prod \mathbb{E}\left(\zeta_{i} \zeta_{j}\right)
$$

which is the desired result. The case where $A$ has an odd number of elements is equally simple.

We note that Theorem 2 can be also easily deduced using generating functions as done in $[3$, Theorem 1.1] who proves a version of Wick's theorem for a Gaussian vector with mean $\mu \neq 0$ : choosing a Bernoulli randomized version of this mean as in (3) yields the result. 


\section{The general case of Gaussian location mixture}

With the useful representation (3), we can generalize the preceding result to any kind of location mixture of Gaussian: namely, we consider a random vector $X \in \mathbb{R}^{d}$ that reads

$$
X=\mu+\zeta
$$

where $\zeta$ is a zero-mean Gaussian vector in $\mathbb{R}^{d}$, independent of the random vector $\mu \in \mathbb{R}^{d}$ with probability distribution $F_{\mu}$; note that the vector $\mu$ may be discrete - taking values $\mu_{i}$ with probabilities $p_{i}$ - or not, but we don't need to assume the existence of a density $f_{\mu}$. In the discrete case, the density of $X$ reads

$$
f_{X}(x)=\sum_{i=0}^{+\infty} p_{i} \phi_{R}\left(x-\mu_{i}\right)
$$

and in the most general case,

$$
f_{X}(x)=\int_{\mathbb{R}^{d}} \phi_{R}(x-\mu) d F_{\mu}(\mu) .
$$

We now state our main theorem.

Theorem 3. Assume that $X \in \mathbb{R}^{d}$ follows model (4) and that all the first-order moments $m_{k}=\mathbb{E} \mu_{k}$ of $\mu$ exist. Then if $A=\left\{\alpha_{1}, \ldots, \alpha_{2 N+\epsilon}\right\}$, with $\epsilon \in\{0,1\}$,

$$
\mathbb{E} X_{A}=\sum_{k=0}^{N} \sum_{\substack{S \subset A \\|S|=2 k+\epsilon}} \mathbb{E}\left(\mu_{S}\right) \sum_{A \backslash S} \prod_{S} \mathbb{E}\left(\zeta_{i} \zeta_{j}\right)
$$

We remark that if all elements $\alpha_{i}$ of $A$ are different and if the vector $\mu$ has independent components, this expression can be further simplified to

$$
\mathbb{E} X_{A}=\sum_{k=0}^{N} \sum_{\substack{S \subset A \\|S|=2 k+\epsilon}}(\mathbb{E} \mu)_{S} \sum_{A \backslash S} \prod_{S} \mathbb{E}\left(\zeta_{i} \zeta_{j}\right),
$$

noting the difference between $\mathbb{E}\left(\mu_{S}\right)=\mathbb{E} \prod_{\alpha_{i} \in S} \mu_{\alpha_{i}}$ in (5) and $(\mathbb{E} \mu)_{S}=\prod_{\alpha_{i} \in S} \mathbb{E} \mu_{\alpha_{i}}$ in (6).

The proof is as follows.

Proof 1. From (4), we deduce

$$
\mathbb{E} X_{A}=\sum_{k=0}^{2 N+\epsilon} \sum_{\substack{S \subset A \\|S|=k}} \mathbb{E}\left(\mu_{S}\right) \mathbb{E}\left(\zeta_{A \backslash S}\right) .
$$

Since the cardinality of $A \backslash S$ is $2 N+\epsilon-k, \mathbb{E}\left(\zeta_{A \backslash S}\right)=0$ unless $|S|=k$ has the same parity as $\epsilon$, in which case it is equal to $\sum_{A \backslash S} \prod_{S} \mathbb{E}\left(\zeta_{i} \zeta_{j}\right)$, hence formula (5). Formula (6) is easily deduced from formula (5) assuming that the components of $\mu$ are independent and that all elements of $A$ are distinct.

We now provide a further generalization of Isserlis theorem by considering a Gaussian vector with both random scale and location parameters. 


\section{A Normal variance-mean mixture application}

The generalized $d$-dimensional hyperbolic distribution was introduced by Barndorff-Nielsen in 1978 [7]. It is the distribution of a random vector that reads

$$
X=\mu+\sigma^{2} \Delta \beta+\sigma \Delta^{\frac{1}{2}} \zeta
$$

where $\mu$ and $\beta$ are two deterministic vectors in $\mathbb{R}^{d}, \Delta$ is a deterministic $(d \times d)$ matrix with $|\Delta|=1$, $\zeta$ is a standard Gaussian vector in $\mathbb{R}^{d}$ and $\sigma^{2}$ is a scalar random variable that follows the Generalized Inverse Gaussian $G I G(\psi, \chi, \lambda)$ distribution

$$
f_{\psi, \chi, \lambda}(x)=\frac{\left(\frac{\psi}{\chi}\right)^{\frac{\lambda}{2}}}{2 K_{\lambda}(\sqrt{\psi \chi})} x^{\lambda-1} \exp \left(-\frac{1}{2}\left(\chi x^{-1}+\psi x\right)\right), x>0
$$

with parameters $\psi>0, \chi>0$ and $\lambda \in \mathbb{R}$. We note that in (7), the GIG random variable $\sigma^{2}$ appears both as a scale and location parameter of the Gaussian vector, hence the "normal variance-mean mixture" name. From the stochastic representation (7), we derive a version of the Isserlis theorem as follows.

Theorem 4. If $X \in \mathbb{R}^{d}$ is a generalized hyperbolic vector as in (7) and $A=\left\{\alpha_{1}, \ldots, \alpha_{2 N+\epsilon}\right\}$ with $\epsilon \in\{0,1\}$ then

$$
\mathbb{E} X_{A}=\sum_{\substack{0 \leq l \leq N \\ 0 \leq p \leq 2 l+\epsilon}} \sum_{\substack{T \subset S \subset A \\|T|=p,|S|=2 l+\epsilon}} \mu_{T} \gamma_{S T T} m_{N+l-p+\epsilon} \sum_{A \backslash S} \prod_{\substack{T \\ 0 \leq 1}} \mathbb{E} Z_{i} Z_{j}
$$

where $\gamma=\Delta \beta$, where $Z$ is a centered Gaussian vector with covariance matrix $\Delta$ and

$$
m_{l}=\mathbb{E} \sigma^{2 l}=\left(\frac{\psi}{\chi}\right)^{-\frac{l}{2}} \frac{K_{\lambda+l}(\sqrt{\psi \chi})}{K_{\lambda}(\sqrt{\psi \chi})} .
$$

Proof 2. Assuming first $\epsilon=0$, we have

$$
\mathbb{E} X_{A}=\mathbb{E} \sum_{l=0}^{N} \sum_{\substack{S \subset A \\|S|=2 l}}\left(\mu+\sigma^{2} \gamma\right)_{S}(\sigma Z)_{A \backslash S}=\sum_{l=0}^{N} \sum_{\substack{S \subset A \\|S|=2 l}} \mathbb{E}\left(\sigma^{2 N-2 l}\left(\mu+\sigma^{2} \gamma\right)_{S}\right) \mathbb{E} Z_{A \backslash S}
$$

with

$$
\mathbb{E} Z_{A \backslash S}=\sum_{A \backslash S} \prod_{S} \mathbb{E} Z_{i} Z_{j}
$$

and

$$
\mathbb{E} \sigma^{2 N-2 l}\left(\mu+\sigma^{2} \gamma\right)_{S}=\sum_{p=0}^{2 l} \sum_{\substack{T \subset S \\|T|=p}} \mu_{T} \gamma_{S \backslash T} \mathbb{E}\left(\sigma^{2}\right)^{N+l-p} .
$$

The moment of order $l$ of the GIG random variable $\sigma^{2}$ can be easily computed from (8) as

$$
m_{l}=\left(\frac{\psi}{\chi}\right)^{-\frac{l}{2}} \frac{K_{\lambda+l}(\sqrt{\psi \chi})}{K_{\lambda}(\sqrt{\psi \chi})},
$$

hence the result. The case $\epsilon=1$ follows the same steps. 


\section{References}

[1] Isserlis L., On a formula for the product-moment coefficient of any order of a normal frequency distribution in any number of variables, Biometrika, 1918, 12, 134-139

[2] Michalowicz J.V., Nichols J.M., Bucholtz F., Olson C.C., A general Isserlis theorem for mixed-Gaussian random variables, Statistics \& Probability Letters, August 2011, $81-8,1233-1240$

[3] Withers C.S., The moments of the multivariate normal, Bulletin of the Australian Mathematical Society, 1985, 32, 103-107

[4] Vignat C. and Bhatnagar S., An extension of Wick's theorem, Statistics \& Probability Letters, 2008, 78-15, 2404-2407

[5] Repetowicz P. and Richmond P., The Wick theorem for non-Gaussian distributions and its application for noise filtering of correlated q-Exponentially distributed random variables, unpublished, arXiv:math-ph/0411020 v1, Nov 2004

[6] Grigelionis B., On the Wick theorem for mixtures of centered Gaussian distributions, Lithuanian Mathematical Journal, 2009, 49-4, 372-380

[7] Barndorff-Nielsen O., Hyperbolic Distributions and Distributions on Hyperbolae, Scandinavian Journal of Statistics, 1978, 5-3, 151-157

[8] Kan R., From Moments of Sum to Moments of Product, Journal of Multivariate Analysis, 99, 542-554, 2008. 